\title{
Blue Stragglers in Galactic Open Clusters and Simple Stellar Population Models
}

\author{
Y. Xin ${ }^{1,2}$ L. Deng ${ }^{2}$ and Z. W. Han $^{1}$ \\ ${ }^{1}$ National Astronomical Observatories/Yunnan, CAS, Kunming, 650011, China \\ emails: xinyu@bao.ac.cn, zhanwen@public.km.yn.cn \\ ${ }^{2}$ National Astronomical Observatories/Beijing, CAS, Beijing, 100012, China \\ email: licai@bao.ac.cn
}

\begin{abstract}
The presence of blue straggler stars (BSs) as secure members of Galactic open clusters (OCs) poses a major challenge to the conventional picture of evolutionary population synthesis (EPS) based on the stellar evolution theory of single stars, since the major formation ways of BSs are all correlated with stellar interactions. With a working sample including 100 Galactic OCs with age ranging from 0.1 to $10 \mathrm{Gyr}$, the contributions of BSs to the integrated light of their host clusters are calculated on an individual cluster base. We also show in this work that the intrinsic evolutionary stages of OCs can be enormously misunderstood by the conventional simple stellar population (SSP) models, if the real/observed integrated spectral energy distributions (ISEDs) of OCs are the only thing that we can depend on and are fitted with ISEDs of conventional SSPs, and consequently the huge uncertainties in age and/or metallicity can be conservatively estimated at $50 \%$. Thus, we strongly confirm that when the conventional EPS technique is used to study the properties of stellar populations in complicated systems such as galaxies, the contributions of BSs should be definitely taken into account in order to avoid big fitting mistake.
\end{abstract}

Keywords. blue stragglers — open clusters and associations: general — Galaxy: stellar content

One of the big challenges to the evolutionary population synthesis (EPS) method is that the effect of complex stellar interactions, such as mass exchanges and coalescence in binaries and collisions between single and/or binaries, is not considered in the popular EPS approaches in the studies of galaxies. The conventional picture of EPS is mainly performed simply based on the evolutionary behavior and spectral libraries of individual stars (Bressan et al. 1994).

We have carried out a study to address this problem semi-empirically, with a big working sample of the Galactic open clusters (OCs), to explore the contributions of blue straggler stars (BSs) to conventional simple stellar population (SSP) models. The main formation mechanisms of BSs are generally related to dynamical interactions of close binaries (Pols \& Marinus 1994) and stellar collisions in high-density regions (Ferraro et al. 2003). Therefore the statistical studies of BS populations in clusters, in terms of their integrated light, provide a clue to stellar interaction processes and the dynamical evolution within a cluster. A full assessment of all these ingredients eventually makes application of the EPS more realistic. To this aim, a large sample of clusters is definitely needed.

The first set of results for old OCs with ages $\geqslant 1$ Gyr was published in Xin \& Deng (2005). An updated set of results includes 100 Galactic OCs with ages $\geqslant 0.1$ Gyr (Xin, Deng \& Han 2007). As general results of this work, the integrated spectral properties of OCs are dramatically modified by the BS content in the clusters. The integrated spectral energy distributions (ISEDs) are greatly enhanced towards shorter wavelengths, becoming significantly bluer, and consequently the broad-band $(U-B)$ and $(B-V)$ colors are modified accordingly. If conventional SSP models are adopted to understand the stellar 

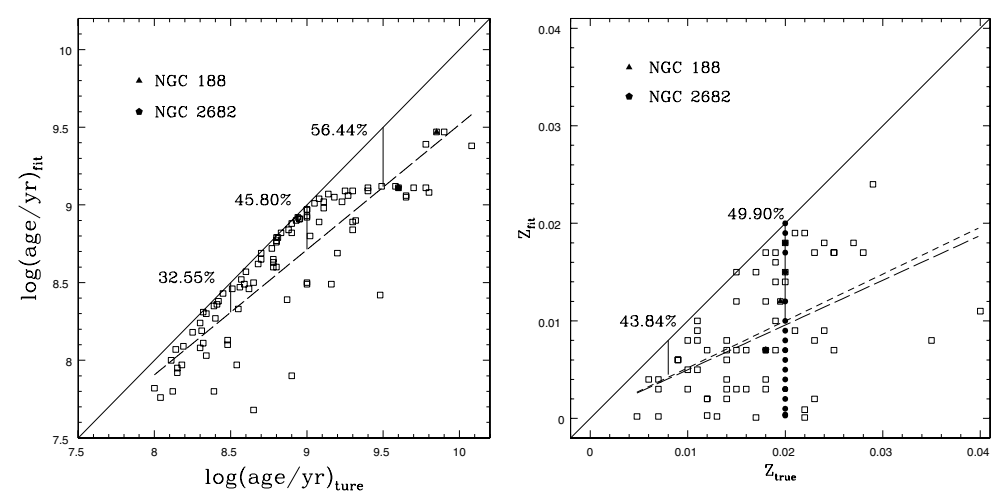

Figure 1. left panel: The uncertainty in the SSP fitting process in terms of cluster ages. The abscissa is the real age of the cluster based on isochrone fitting results from the literature. The ordinate is the fitted age based on the conventional SSP model. Open squares show the results for all 100 clusters in the sample. The results for NGC 188 and NGC 2682 (M67) are highlighted by the solid triangle and solid pentagon, respectively. Differences between the points and the solid line represent the uncertainty in age when using conventional SSP models. The dashed line is the least-square fit result for the open squares. right panel: The uncertainty in the SSP fitting process in terms of cluster metallicity. Open squares show the results for sample clusters having published metallicity. Solid circles show the results for sample OCs for which we assumed $\mathrm{Z}=0.02$. Differences between the points and the solid line represent the uncertainty in metallicity when using the conventional SSP models. The short-dashed line is the least-squares fit result for the open squares. The long-dashed line is the least-squares fit result for all points.

content in galaxies, either using spectra or broad-band colors, significant uncertainties in age and/or metallicity will be introduced. In the parameter ranges covered by our sample OCs (Figure 1), the age underestimation (identified as $\left(\right.$ age $_{\text {true }}$-age $_{\text {fit }}$ )/age true $_{\text {f }}$ ) amounts to $30 \%$ at $\log ($ age $/ \mathrm{yr})=8.5,45 \%$ at $\log ($ age $/ \mathrm{yr})=9.0$ and $55 \%$ at $\log ($ age $/ \mathrm{yr})=9.5$. There is a trend for the rates to become larger for greater ages. The metallicity parameter can also be seriously underestimated by using conventional SSP models. This is true at least qualitatively within the scope of the current work. Due to the uncertainties in measuring the metallicities of star clusters, accurate results can not be given.

Taking OCs as general representatives of SSPs in galaxies, and limiting our results to the age and metallicity ranges covered by the working sample in the current work, the conventional SSP models derived from the single star evolution theory are seriously altered by the descendants of stellar interactions that are presumably common in dense environments. Given the BSs are common in all stellar systems, their contributions to ISEDs of the embedded populations must be taken into account when the EPS technique is applied to study the properties of stellar populations in galaxies.

\section{Acknowledgements}

We would like to thank the Chinese National Science Foundation for support through grants 10573022, 10333060, 10521001 and 10433030.

\section{References}

Bressan, A., Chiosi, C. \& Fagotto, F. 1994, ApJS 94, 63

Ferraro, F.R., Sills, A., Rood, R.T., Paltrinieri, B., \& Buonanno, R. 2003, ApJ 588, 464

Pols, O.R. \& Marinus, M. 1994, A\&A 288, 475

Xin, Y. \& Deng, L. 2005, ApJ 619, 824

Xin, Y., Deng, L. \& Han, Z.W. 2007, ApJ accepted, astro-ph/0609745 